\title{
Bi-Directional Effects of Stimulus Vertical Position and Construal Level
}

\author{
Ravit Nussinson ${ }^{1,2}$, Yaron Elias ${ }^{1}$, Sari Mentser ${ }^{1}$, Yoav Bar-Anan $^{3}$, and Nurit Gronau ${ }^{1}$ \\ ${ }^{1}$ Department of Education and Psychology, The Open University of Israel, Raanana, Israel \\ ${ }^{2}$ Institute of Information Processing and Decision Making, The University of Haifa, Israel \\ ${ }^{3}$ Department of Psychology, Ben-Gurion University of the Negev, Israel
}

\begin{abstract}
We suggest that there is an association in people's minds between the vertical position of a stimulus (up vs. down) and its construal level (high vs. low), which results in bi-directional effects between the dimensions. In Study 1, participants exhibited both implicit and explicit associations between the dimensions. Studies 2 and 3 demonstrated that stimulus construal level affects its vertical position, with participants showing a preference for positioning abstract concepts higher up and concrete concepts lower down. Study 4 testified to the effect of vertical positioning of information on its level of construal. Behaviors presented at the top of a display (more than those presented at the bottom) were construed in terms of why they are performed rather than how to perform them. Theoretical and practical implications are discussed.
\end{abstract}

Keywords: construal level, vertical position, distance, up, down, association

People experience the world as a set of construals. The neighbor's cat mewing in the backyard, the memory of an old insult, an upcoming organizational event that we are responsible for coordinating, and indeed, our very selves all exist as mental representations which populate our minds and which serve as the foundations of our interactions with the world. However, our mental representations vary with respect to their level of construal. Some representations, those of a high-construal level, are abstract, coherent, and superordinate. They focus on the gist of the stimulus (e.g., the object, person, or event) and on its invariable features. Other representations, those of a low-construal level, are concrete, less coherent, and subordinate. They focus on less central, more incidental features of the stimulus and are hence more contextualized (Liberman \& Trope, 1998, 2008; Trope \& Liberman, 2010). Importantly, construal is a function of the person's mental representation at the time and not some inherent feature of the stimulus. Indeed, the very same object or event may be construed at a high level on one occasion and a low level on another. For example, a target action (e.g., making a list) may be construed in terms of the goal toward which it is directed (getting organized - an abstract goal, and therefore a high-construal level) or in terms of the means by which it is performed (writing things down - a concrete act, and therefore a low-construal level) (Vallacher \& Wegner, 1987).
The current research suggests the existence of an association between construal level and the positioning of a stimulus along the vertical dimension in space, such that up and down are associated with high- and low-construal levels, respectively. In what follows, we delineate the rationale underlying this hypothesis.

\section{Construal Level and Psychological Distance}

Recent theorizing suggests that a key function of mental representations, with their varied levels of construal, is to allow people to traverse psychological distance (Trope \& Liberman, 2010). Specifically, our direct experience is limited to the self, here and now. Still, to function in the world, we must be able to think about physically distant places (spatial or physical distance), to plan ahead into the future (temporal distance), to take the perspective of others (social distance), and to imagine unlikely scenarios (hypotheticality). Ample evidence suggests that people use increasingly higher levels of construal to represent objects as psychological distance from the object increases. For example, when a moment of decision (e.g., choosing a career path or vocation) is temporally distant, people attribute relatively more importance to considerations involving abstract 
superordinate goals (e.g., the ability to derive personal joy) than when the moment is temporally close. Conversely, when the moment is temporally close, people attribute more importance to considerations involving concrete subordinate goals (e.g., the ability to work under comfortable conditions) (Elias, Nussinson, \& Roccas, 2018).

It is assumed that high-level construals are useful in representing psychologically distant objects precisely because these goals are more likely to remain unchanged as one gets closer to or further from an object (Trope \& Liberman, 2010). Low-level construals are useful in representing psychologically close objects because they provide us with the more detailed and specific information needed to interact with them. Hence, "the different levels of construal serve to expand and contract one's mental horizons and thus mentally traverse psychological distances" (ibid., p. 442).

\section{Vertical Position, Physical Distance, and Construal Level}

Importantly, evidence suggests that the ecology of the world that we live in results in a correlation between at least one dimension of psychological distance - physical distance and the vertical dimension of space. In natural environments, nearby objects are more prevalent in the lower part of the visual field, while distant objects are more prevalent in the upper part of the visual field. Even the ground surface seems to rise upward from near (the spot where your feet rest) to far (the horizon; Ooi, Wu, \& He, 2001). This link between physical distance and location in the visual field holds for indoor as well as outdoor environments (Bruno \& Cutting, 1988; Cutting \& Vishton, 1995; Previc, 1998; Previc, Declerck, \& de Brabander, 2005; Yonas, Elieff, \& Arterberry, 2002). Indeed, objects that lie physically within our reach are usually located below eye level (Previc, 1990).

The cognitive system seems to be tuned to this regularity of our environment. As succinctly summarized by Gibson: "'Upness' is ... a fairly reliable cue to the distance of an object in the visual field ... Of two objects in a perfectly blank frame, the upper will appear to be farther away" (Gibson, 1950, p. 180). In line with this regularity, findings suggest that the positioning of a stimulus along the vertical dimension affects its perceived distance from the observer (Li \& Guo, 1995; Ooi, Wu, \& He, 2001). Similarly, upward versus downward head and eye movements result in increased versus decreased estimates of physical distance, respectively (Van Kerckhove, Geuens, \& Vermier, 2014).

As noted above, numerous findings suggest that physically distant objects are represented using high-level construals, while physically close objects are represented using low-level construals (Amit, Algom, \& Trope, 2009; Bar-Anan, Liberman, \& Trope, 2006). If objects that are higher in our field of view are typically more distant and objects that are lower are typically close, it follows that we can expect objects which are higher in the visual field ("up" or "above") to be represented on a high-construal level and objects which are lower in the visual field ("down" or "below") to be represented on a low-construal level. We thus hypothesize an association between the vertical positioning of a stimulus and its construal level.

Some previous findings support this line of reasoning. For instance, in Meyers-Levy and Zhu (2007), rooms with high ceilings were shown to activate concepts related to freedom, which primes abstraction, whereas rooms with low ceilings were shown to activate concepts related to confinement, which primes concreteness. Similarly, Van Kerckhove et al. (2014) found that participants who performed upward head or eye movements preferred more abstract restatements of activity descriptions, assigned products to broader, more inclusive categories, preferred desirable over feasible products, and demonstrated less preference-decision consistency than participants whose heads or eyes were directed forward, in a neutral position. The reverse pattern was obtained for participants who engaged in downward head or eye movements. However, while such findings are suggestive of a possible link between vertical position and construal level, no studies, to our knowledge, have focused specifically on the congruence between the vertical positioning of a stimulus and its construal level. Moreover, if indeed there is an association between stimulus' vertical position and its construal level, this may result in two kinds of effects: an effect of stimulus construal level on its preferred vertical position and an inversed effect of stimulus vertical position on its preferred construal level (see Cian, Krishna, \& Schwarz, 2015, for a similar rationale with respect to the association between vertical position and rationality/emotionality).

The present research examines these questions directly, in four studies. In Study 1, we lay the groundwork, by testing for the existence of both implicit and explicit mental associations between the two dimensions. Studies 2-4 explore the two possible effects of this association. In Studies 2 and 3, we empirically examine the effect of stimulus construal level on vertical positioning, testing whether people prefer to position words representing abstract (highconstrual) concepts above words representing concrete (low-construal) concepts on a computer screen. In Study 4 , we consider the opposite effect - that is, the effect of relative vertical position on level of construal. We examined whether the positioning of text at the top or bottom of a card affected whether participants were more likely to construe the text at a high or low level (specifically, in terms of why or how an action is performed, respectively; Fujita, Henderson, Eng, Trope, \& Liberman, 2006; Vallacher \& Wegner, 1987, 1989). 


\section{Study 1: Do People Associate Construal Level With Vertical Position?}

This study empirically examines whether people implicitly and explicitly associate words conveying abstractness (e.g., "abstract," "broad") with words conveying the concept up (e.g., "above," "upper") and words conveying concreteness (e.g., "concrete," "particular") with words conveying the concept down (e.g., "below," "lower"). To measure an implicit association between the two dimensions (abstractconcrete and up-down), participants completed a Sorting Paired Features task (SPF; Bar-Anan, Nosek, \& Vianello, 2009). Like the Implicit Association Test (IAT; Greenwald, McGhee, \& Schwartz, 1998), the SPF measures mental associations between concepts. However, the IAT involves right-left sorting of both dimensions, which may interact with our up-down dimension (see Cho \& Proctor, 2003). In contrast, vertical positioning is inherent to the SPF (one of the dimensions is organized vertically on the display), rendering it especially potent for examining mental associations involving the vertical dimension. Furthermore, the SPF measures all the associations in the same block, thus eliminating the block-order effects often found with the IAT (Greenwald et al., 1998).

To measure an explicit association between the two dimensions, participants indicated how strongly they associate concepts from the two dimensions on visual analog scales (VAS). We examined whether participants explicitly associated construal level (abstract vs. concrete) with vertical position (up vs. down, respectively) and whether they associated vertical position with construal level (the association in the reverse direction).

While we hypothesized that the automatic mental association between the two dimensions would be reflected in performance on the SPF test, we were not sure whether participants would be conscious of these associations and able to explicitly report on them. Thus, we did not have strong predictions as to participants' performance on the explicit measures.

\section{Method}

In all are studies, we report how we determined sample size, all data exclusions, all manipulations, and all measures.

\section{Participants}

Participants were recruited from Project Implicit's participant pool (http://implicit.harvard.edu; Nosek, 2005). To maximize the odds of obtaining our hypothesized effect, we conducted a power analysis aimed at $99.9 \%$ power to detect a small effect $(d=0.25)$. This analysis indicated that a sample size of 400 participants was required. Volunteers were randomly assigned to this study from a pool of available studies. Six hundred seventy-four participants consented to participate, and 405 completed the SPF task (the high dropout rate is typical of Project Implicit because participants are mainly motivated by curiosity and do not receive compensation for their participation).

Following Bar-Anan et al. (2009), of the 405 participants who completed the SPF, we excluded 25 participants $(6.2 \%$ of the sample) who responded either too quickly (RT < 400 ) or too slowly (RT $>5,000$ ) in more than $10 \%$ of the trials. This left 380 participants (215 females, $M_{\text {age }}=$ $37.9, S D=14.00)$ in the SPF analyses. A total of $379(213$ females, $M_{\text {age }}=37.8, S D=14.2$ ) participants responded to all eight explicit association questions and were included in the explicit associations analyses (182 rated vertical positioning words, 197 rated construal-level words). About 7\% of the participants were Black, about $8 \%$ were Asian and, the rest were of other or mixed racial origin (ethnic distribution among participants completing the SPF task and those completing all explicit association questions was similar). About $35 \%$ of the participants reported that they are the citizens of a country other than the United States. The results are the same if we include only the 363 participants who were not excluded from either the implicit or the explicit analyses (the supplementary materials of Study 1 are available at https://osf.io/ky25r/).

\section{Materials and Procedure} Implicit Association Measure

In the SPF, participants categorized pairs of words (e.g., general + above) into one of four pairs of categories (Abstract + Up, Abstract + Down, Concrete + Up, Concrete + Down) in three blocks of 40 trials (see Figure 1). Each word pair included one of four words representing abstractness (abstract, broad, general, universal) or concreteness (concrete, particular, specific, narrow) and one of four words representing up (above, upper, top, over) or down (below, lower, bottom, under). Faster response times for categorizing word pairs from one category pair (e.g., Abstract + Up) compared to another (e.g., Abstract + Down) can be interpreted as indicating stronger associations for the former than for the latter (i.e., an implicit association between words reflecting the concept $u p$ and the concept abstract).

Following standard procedure for computing scores for the SPF in the context of social groups (Bar-Anan et al., 2009), we examined whether participants preferred word pairs that matched the combinations Abstract + Up and Concrete + Down over pairs that matched Abstract + Down and Concrete + Up. Specifically, we first computed the difference between average response latency in trials where the target word pairs matched either of the combinations 
(A)

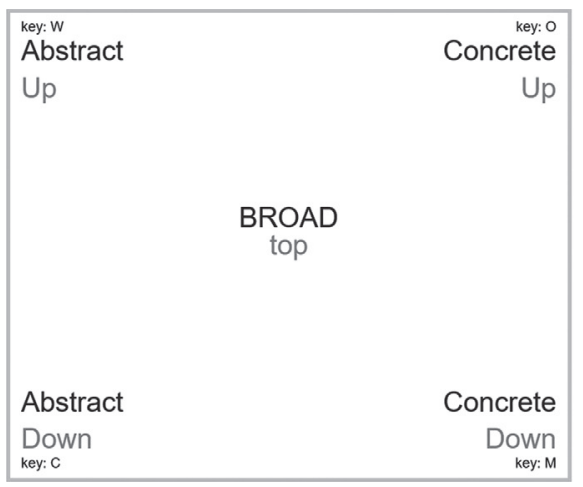

(B)

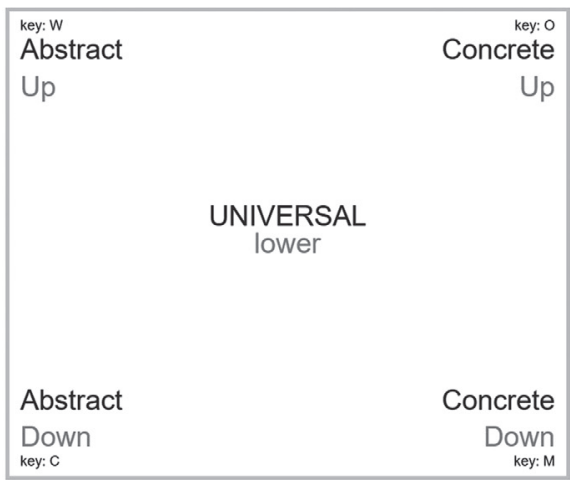

Figure 1. SPF trials for example: (A) congruent trial; (B) incongruent trial (Study 1).
Abstract + Up or Concrete + Down and the average response latency in trials where the match was to Abstract + Down or Concrete + Up. This difference was computed for each of the three SPF blocks, then divided by the overall standard deviation of that block to produce that block's SPF $D$ score. The average of the three SPF $D$ scores was the overall SPF $D$ score, used as the measure of implicit associations. Positive SPF $D$ scores indicated stronger associations for the combinations Abstract + Up and Concrete + Down than for the combinations Abstract + Down and Concrete + Up.

In the SPF, each of the four category combinations is located in the corner of a square, such that the categories belonging to one dimension are separated vertically and those belonging to the other dimension are separated horizontally (see Figure 1). In this study, combinations containing the category Up were always positioned in the two top corners of the square, and combinations containing Down in the bottom corners. We manipulated between participants whether the categories Abstract and Concrete appeared in the left- and right-hand corners, respectively, or vice versa. The $\mathrm{W}$ and $\mathrm{C}$ keys were used to indicate the upper and lower left corners, respectively, and the $\mathrm{O}$ and $\mathrm{M}$ keys the upper and lower right corners. Participants were asked to put their left pinky and index fingers on the $\mathrm{W}$ and $\mathrm{C}$ keys (respectively), and they were asked to put their right index and pinky fingers on the $\mathrm{O}$ and $\mathrm{M}$ keys (respectively).

\section{Explicit Association Measure}

Following the SPF, participants completed one of two selfreport questionnaires. Half the participants were given each of the eight words used for the concepts Abstract and Concrete (i.e., abstract, broad, general, universal, concrete, particular, specific, narrow) and asked to indicate for each one whether they associated that word more with the concept Up or the concept Down (e.g., "Do you associate the concept 'specific' more with the concept 'Up' or the concept 'Down'?'). They indicated their answers using a visual analog scale (VAS) that ranged from Up to Down (we randomly assigned one of the concepts to the left side of the VAS and the other to the right, with the label Equal Association at the center; see Figure 2). The other half of the participants received a questionnaire in which the rated words were the eight words used for the concepts Up and Down (i. e., above, upper, top, over, below, lower, bottom, under) and the scale ranged from Abstract to Concrete (again, randomly assigned to the left and right with Equal Association at the center; Figure 2). The two versions of the questionnaire were randomized between participants. Responses were coded with a number from 0 to 100 , and recoded such that larger numbers indicated a stronger association with Up or Abstract than with Down or Concrete.

\section{Results and Discussion}

The dataset is available at https://osf.io/ky25r/.

\section{Implicit Associations}

As hypothesized, the mean SPF score was 0.201 ( $S D=$ $0.443)$, significantly different from zero, $t(379)=8.829$, $p<.001, d=0.453,95 \%$ CI $[0.348,0.559]$, indicating stronger associations between Abstract and Up and between Concrete and Down than between Abstract and Down and between Concrete and Up. The location of the construal-level concepts (i.e., whether Abstract was to the left or the right) had no significant effect on the SPF scores, $t(372.06)=0.904, p=.366, d=0.093,95 \%$ CI [0.008, 0.193].

\section{Explicit Associations}

As noted above, responses to the explicit association measures were coded such that larger numbers indicated a stronger association with Up (rather than Down) or Abstract (rather than Concrete). Thus, our results reflect a relative association with Up as compared to Down and with Abstract as compared to Concrete, rather than an absolute association with each of these concepts separately. 
(A)

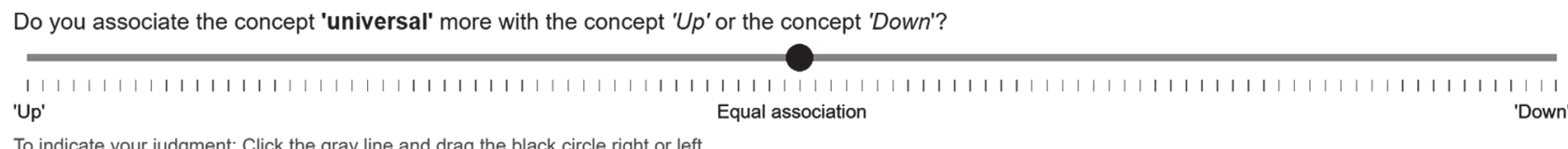

To indicate your judgment: Click the gray line and drag the black circle right or left.

(B)

Do you associate the concept 'bottom' more with the concept 'Abstract' or the concept 'Concrete'?

'Abstract'
To indicate your judgment: Click the gray line and drag the black circle right or left.

Figure 2. Explicit associations questions for example: (A) a question measuring association with Up versus Down; (B) a question measuring association with Abstract versus Concrete.

Table 1. Means and standard deviations of explicit mental associations

\begin{tabular}{|c|c|c|c|c|c|c|}
\hline \multirow[b]{3}{*}{ Abstract words } & \multicolumn{6}{|c|}{ Associations with Up } \\
\hline & \multicolumn{2}{|c|}{ Up on the left $(n=98)$} & \multicolumn{2}{|c|}{ Down on the left $(n=99)$} & \multicolumn{2}{|c|}{ Total $(n=197)$} \\
\hline & $M=56.556$ & $S D=14.992$ & $M=57.083$ & $S D=15.548$ & $M=56.821$ & $S D=15.238$ \\
\hline \multirow[t]{3}{*}{ Concrete words } & $M=51.031$ & $S D=14.650$ & $M=45.114$ & $S D=17.146$ & $M=48.057$ & $S D=16.186$ \\
\hline & \multicolumn{6}{|c|}{ Associations with Abstract } \\
\hline & \multicolumn{2}{|c|}{ Abstract on the left $(n=101)$} & \multicolumn{2}{|c|}{ Concrete on the left $(n=81)$} & \multicolumn{2}{|c|}{ Total $(n=182)$} \\
\hline Up words & $M=50.693$ & $S D=18.318$ & $M=47.738$ & $S D=17.920$ & $M=49.378$ & $S D=18.152$ \\
\hline Down words & $M=46.943$ & $S D=16.966$ & $M=46.290$ & $S D=16.508$ & $M=46.652$ & $S D=16.721$ \\
\hline
\end{tabular}

For those questionnaires where the construal-level words (reflecting abstractness and concreteness) were rated against the vertical positioning concepts (Up and Down), we submitted the average reported associations (detailed in Table 1) to a 2 (construal level: Concrete, Abstract) $\times 2$ (concept on the left of the VAS: Down, Up) mixed analysis of variance (ANOVA). A main effect of construal level indicated that as hypothesized, participants associated Abstract concepts more than Concrete concepts with the category Up, $F(1,195)=19.683, p<.001, \eta_{p}^{2}=.092,95 \%$ CI $[.037, .160]$. In addition, participants associated the concepts more with Up when Up was on the right side of the VAS than when it was on its left side, $F(1,195)=6.820, p$ $=.010, \eta_{\mathrm{p}}^{2}=.034,95 \%$ CI $[.005, .085]$. There was no significant interaction, $F(1,195)=2.671, p=.104, \eta_{\mathrm{p}}^{2}=.014$, $95 \%$ CI $[.000, .052]$. Thus, the results indicate that participants explicitly associate abstractness more than concreteness with the category Up (rather than Down).

For those questionnaires where the vertical positioning words were rated against the construal-level concepts (Abstract and Concrete), we submitted the average reported associations (detailed in Table 1) to a 2 (vertical position: Up, Down) $\times 2$ (concept on the left of the VAS: Abstract, Concrete) mixed ANOVA. There was no effect of vertical position category, $F(1,180)=1.893, p=.171$, $\eta_{\mathrm{p}}^{2}=.010,95 \%$ CI $[.000, .048]$, VAS direction, $F(1,180)$ $=1.333, p=.250, \eta_{\mathrm{p}}^{2}=.007,95 \%$ CI [.000, .041], or interaction, $F(1,180)=0.262, p=.650, \eta_{p}^{2}=.001,95 \% \mathrm{CI}$ $[.000, .024]$. Thus, there was no evidence of an explicit association between vertical positioning and construal level in participants' ratings of words used to denote vertical position.

In sum, supporting our hypothesis, the results suggest that people automatically associate construal level (abstractness vs. concreteness) and relative vertical position (up vs. down). They further suggest that people are at least somewhat aware of this association, though only in one direction: People explicitly associate words reflecting abstractness (rather than concreteness) with the concept "up" (rather than "down"), but they do not consciously associate words reflecting higher vertical position with abstractness. This asymmetry may reflect the fact that vertical position is a salient physical dimension of stimuli in the environment. Hence, people commonly use vertical position to conceptualize abstractness and concreteness (as, indeed, is evident in the very terms high-and low-construal level), but do not need abstractness and concreteness to conceptualize vertical position. 


\section{Study 2: The Effect of Construal Level on Vertical Positioning - Abstract and Concrete Concepts}

Study 1 examined the semantic association between the two dimensions - construal level and vertical positioning. In Studies 2 and 3, we sought to examine the derived effect of one of the dimensions on the other.

In Study 2, we asked participants to position each of two words, one reflecting an abstract word and the other a concrete word, in boxes located at the top and bottom of a vertically oriented rectangle, following their "gut feeling" (Figure 3). We hypothesized that a spatial organization that is congruent with people's daily experience (abstract up, concrete down) would be intuitively appealing to participants. We hence expected participants to position the abstract word at the top of the rectangle and the concrete word at the bottom with a probability greater than chance.

\section{Method}

\section{Participants}

To compute the required sample size, we conducted a power analysis using $G^{*}$ Power software (Faul, Erdfelder, Lang, \& Buchner, 2017). Based on the less than medium effect obtained in Study 1 and the substantial difference between Study 1 and the present study, we aimed at a power of $80 \%$ to detect a small-to-medium effect $(d=$ 0.35 ) in a one-sample $t$-test. This analysis suggested recruiting a total of 67 participants. Because the study was conducted online and we expected "noisy" running conditions, we recruited a sample of 88. All participants were Hebrew-speaking Israeli students (52 females, $M_{\text {age }}$ $=27.19, S D=4.21)$. The study was conducted in Hebrew.

\section{Materials and Procedure}

Participants were asked to first press F11 so as to view the study on a full screen. They were then presented with a vertically oriented rectangle containing two small boxes, one at the top of the rectangle and the other at the bottom. They were asked to ensure that they could view all four sides of the rectangle without having to scroll down (they were encouraged to reduce the size of the display by simultaneously pressing Ctrl and - if needed).

In the instructions, participants were told that the task would involve intuitive processing of spatial information. It was explained that they would be presented (on their

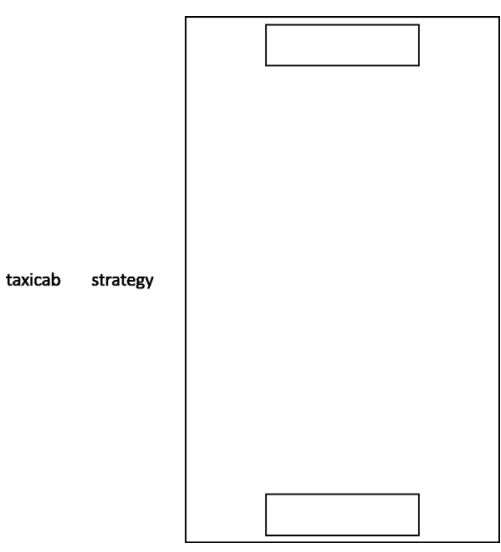

Figure 3. An example trial used in Study 2.

screens) with vertical cards (the vertically oriented rectangles described above) along with a pair of words. Their task would be to position the words by dragging and dropping them, one in the box located at the top of the card and the other in the box located at the bottom, so that the organization of the words "felt right" to them (see Figure 3). ${ }^{1}$ Participants were ensured that there were no right or wrong answers in this task and were encouraged to follow their gut feelings.

Participants were presented with a total of 30 pairs of words. In each pair, one word represented an abstract concept (e.g., "custom," "meaning") and the other a concrete object (e.g., "hut," "taxicab") (see Table A1 in the Appendix). The words were chosen based on the concreteness ratings published by Brysbaert, Warriner, and Kuperman (2014); the concreteness ratings of the concrete words were significantly higher than those of the abstract words, $t(29)=28.754, p<.001$. In selecting the words, we ensured that the concrete and abstract words did not differ in valence (based on norms by Warriner, Kuperman, \& Brysbaert, 2013) ${ }^{2} t(29)=.431, p=.670, \mathrm{BF}_{01}=4.720$, nor in their frequency in the Hebrew language, $t(29)=.518, p=$ $.609, \mathrm{BF}_{01}=4.544$ (based on Frost \& Plaut, 2005). The two words were presented side by side to the right of the rectangular card, midway up. ${ }^{3}$ Location of the abstract word (to the right or to the left of the concrete word) was counterbalanced across participants.

\section{Results and Discussion}

The dataset is available at https://osf.io/ky25r/.

If participants positioned abstract and concrete words randomly at the top or bottom of the card, we would expect to find the word pairs displayed in congruence with the

\footnotetext{
1 Figure 3 presents an example of the task using words in English. As noted above, the actual experiment was conducted in Hebrew.

${ }^{2}$ We could not find Hebrew norms for valence and concreteness level and so based these on the English translations of the chosen words.

${ }^{3}$ In Figure 3, the words are shown on the left of the rectangular card because English is written from left to right. Hebrew is written from right to left. Thus, the words in this task are presented such that the eye naturally moves from the words to the card, rather than the other way around.
} 
hypothesis (i.e., abstract word up and concrete word down) in $50 \%$ of the trials. However, if participants associated abstractness with up and concreteness with down, we would expect to find the word pairs positioned in congruence with the hypothesis more than $50 \%$ of the time. In our sample, the proportion of pairs placed in congruence with the hypothesis was $.710(S D=0.230)$. A $t$-test comparing this proportion with the constant 0.5 yielded a significant difference, $t(87)=8.584, p<.001, d=0.915,95 \%$ CI [0.664, 1.162]. Thus, the results of Study 2 support our assumption that high- and low-construal levels are indeed congruent with up and down, respectively, by demonstrating an effect of stimulus construal level on vertical positioning.

\section{Study 3: The Effect of Construal Level on Vertical Positioning: Categories and Exemplars}

Categories are broad, general, and abstract mental representations (e.g., drinks), while exemplars serve as specific instances (e.g., orange juice) (Fujita et al., 2006; Trope \& Liberman, 2010; Wakslak \& Trope, 2009). Study 3 was identical to Study 2 except that instead of words for abstract and concrete concepts, participants were asked to position a category and its exemplar in boxes located at the top and bottom of a vertically oriented card on their screens. Here, too, we hypothesized that a spatial organization of the words that is congruent with people's daily experience, namely abstract (category) up and concrete (exemplar) down, would be intuitively appealing to participants. We hence expected participants to position the category at the top of the card and the exemplar at the bottom with a probability greater than chance.

\section{Method}

This study was preregistered at AsPredicted.Org, https:// aspredicted.org/blind.php?x=y8up7g.

\section{Participants}

We conducted a power analysis similar to that of Study 2 guided by similar considerations. The analysis suggested we would need 67 participants. Because the study was conducted online and we expected "noisy" running conditions, we recruited 93 participants. All participants were Hebrewspeaking Israeli students (61 females, $M_{\text {age }}=28.77$, $S D=4.13)$.

\section{Materials and Procedure}

To accentuate the difference in level of abstractness between categories and exemplars, we used superordinate

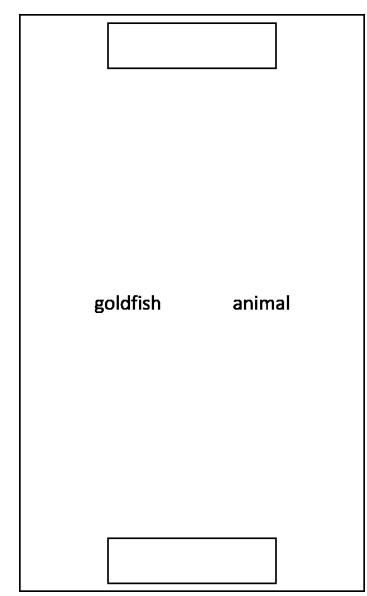

Figure 4. An example trial used in Study 3.

concepts for the categories (e.g., furniture, communication device, plant) and subordinate concepts for the exemplars (e.g., wooden chair, iphone, anemone) (Markman \& Wisniewski, 1997; McCloskey \& Zaragoza, 1985; Pansky \& Tenenboim, 2011; Rosch, Mervis, Gray, Johnson, \& Boyes-Braem, 1976). This time, the two words were presented side by side in the center of the rectangular card, midway up (see Figure 4). Again, the order of the two words (category to the right or left of the exemplar) was counterbalanced across participants.

\section{Results and Discussion}

The dataset is available at https://osf.io/ky25r/.

As in Study 2, we conducted a one-sample $t$-test comparing the proportion of word pairs positioned in congruence with the hypothesis with the proportion expected by chance (i.e., 50\%). This analysis confirmed that the average proportion of pairs in our sample positioned in congruence with the hypothesis $(M=0.780, S D=0.319)$ was significantly larger than $0.5, t(92)=8.483, p<.001, d=0.880$, 95\% CI [0.638, 1.117].

The results of Study 3 conceptually replicate those of Study 2 and support our assumption that high- and low-construal levels are indeed congruent with up and down, respectively, by demonstrating an effect of stimulus construal level on vertical positioning.

\section{Study 4: The Effect of Vertical Positioning on Stimulus Construal Level}

If indeed congruence between the vertical positioning of a stimulus and its construal level prevails, then high 
construals of a stimulus should be relatively easier to process when presented in a higher position (e.g., on a screen or page), while stimuli at a low-construal level should be relatively easier to process when presented in a lower position (see Cian et al., 2015, for a similar rationale with respect to the association between vertical position and rationality/ emotionality). Thus, when given the choice between construing a stimulus at a high or low level, people should be more likely to choose a high-construal level when the stimulus is presented in a position that suggests "up" rather than "down." Study 4 empirically tested this hypothesis.

According to Vallacher and Wegner's $(1987,1989)$ action identification theory, actions (e.g., conducting a study) may be represented in terms of superordinate goals, associated with the end for which we perform the action (e.g., advancing science), or in terms of subordinate goals, associated with the means by which we perform it (e.g., testing a hypothesis). The first, high-level action identification is associated with "why" we perform an action, whereas the latter, low-level action identification is associated with "how" we perform it. Ample evidence suggests that psychological distance (proximity) renders high- (low-)level construals of actions more accessible (Day \& Bartels, 2004; Fujita et al., 2006; Libby, Shaeffer, \& Eibach, 2009; Liberman \& Trope, 1998; Liviatan, Trope, \& Liberman, 2008; Wakslak, Trope, Liberman, \& Alony, 2006). In Study 4, we examined whether the vertical position at which the name of an action was presented (at the top or bottom of a display) affected whether it was construed in terms of the ends for which it is performed (the why) or the means by which it is performed (the how).

Participants were presented with common behaviors (e. g., opening a door) expressed in a few words printed at either the top or the bottom of a vertically oriented card. For each behavior, participants indicated which of two alternative descriptions or action identifications best fit the behavior: a high-level action identification or a low-level action identification. If congruence exists between how people construe a behavior and its vertical positioning, then participants should prefer high-level action identifications to a greater extent for actions printed at the top of the card compared with the bottom, and low-level action identifications to a greater extent for actions printed at the bottom of the card compared with the top.

\section{Method}

\section{Participants}

To compute the required sample size, we conducted a power analysis using $G^{*}$ Power software. We aimed to obtain a power of $80 \%$, which would allow us to detect a medium effect $\left(\eta_{p}^{2}=.06\right)$ in a repeated-measures ANOVA. This assumed effect size was based on the relatively large effects found in Studies 2 and 3 while recognizing the more delicate nature of the current experiment. The analysis suggested recruiting a total of 126 participants. In spite of our efforts, we were only able to recruit a total of 109 participants for this study. All were Hebrew-speaking IDF soldiers who volunteered to participate in the study. Seventy-four were male; 35 were female. Their mean age was 29.62 , $S D=11.19$.

\section{Materials and Procedure}

Participants were told that every action can be described in various ways. For example, the behavior typing a document might be described as pressing down keys or as expressing thoughts. In each trial, participants were presented with a set of stimuli comprising a short action together with two descriptions (a total of 22 sets borrowed from Vallacher \& Wegner's [1989] Behavior Identification Form, which were translated into Hebrew). One of the descriptions in each set was at a high-construal level, focusing on the end for which the action is performed (the why), and the other was at a low-construal level, focusing on the means by which the action is performed (the how). The participants' task was to quickly and intuitively choose the description which best fit the action.

In this experiment, physical stimuli were used (rather than words on a screen). The actions were printed at either the top or the bottom of a vertically oriented plastic card $(297 \mathrm{~mm} \times 210 \mathrm{~mm}), 132 \mathrm{~mm}$ above or below the center of the card, and the two descriptions were printed to the right and left of the action. The cards were inserted into a simple apparatus which allowed for their vertical presentation. If participants chose the right-hand description as best fitting the action, they pulled out the card and placed it facing down to the right of the apparatus. If they chose the left-hand description, they placed the card to the left of the apparatus. The vertical positioning of each action and the location of each of the two descriptions (to the right or left of the action) were counterbalanced between participants, resulting in four versions of the experiment. Participants were randomly assigned to one of the four versions.

Participants sat at a distance of $50 \mathrm{~cm}$ from the cards, and participants' seats adjusted so that participants' eye level was at the center of the card, requiring participants to tilt their gaze upward or downward at about $15^{\circ}$ to view the stimuli.

\section{Results and Discussion}

The dataset is available at https://osf.io/ky25r/.

For each participant the proportion of high-construal description chosen as best fitting, the action was computed separately for sets printed at the top versus the bottom of the card. A Vertical Position (Up vs. Down) $\times$ Version 
(1-4) mixed ANOVA with the first variable as a within-participant factor and the second as a between-participant factor found no effect of version on the proportion of highconstrual descriptions chosen, $F(3,105)=0.646, p=.587$, $\eta_{\mathrm{p}}^{2}=.018,95 \%$ CI $[.000, .069]$. In addition, version did not interact with the vertical position of the actions and descriptions, $F(3,105)=1.270, p=.288, \eta_{p}^{2}=.035,95 \%$ CI $[.000, .103]$. The results showed that a greater proportion of high-construal descriptions were chosen when the materials were printed at the top of the card $(M=0.726$, $S D=0.204)$ compared with the bottom $(M=0.582, S D=$ $0.309), F(1,105)=28.258, p=.0001, \eta_{\mathrm{p}}^{2}=.212,95 \% \mathrm{CI}$ [.088, .338]. The proportion of low-construal descriptions chosen in each case was, of course, complementary $(M=$ $0.274, S D=0.204$ for sets printed at the top; $M=0.418$, $S D=0.309$ for sets printed at the bottom). Thus, supporting our hypothesis, participants preferred high-level action identifications to a greater extent when the actions and descriptions were presented at the top of a card (compared with the bottom of the card), and they preferred low-level action identifications to a greater extent when these were presented at the bottom of the card (compared with the top).

Note that even when the actions and descriptions were printed at the bottom of the cards, the percentage of high-construal responses was not lower than 50\% ( $M=$ $0.582, S D=0.309)$. This implies that regardless of the presentation format, participants did not tend to construe the behaviors in terms of their means. This result goes hand in hand with previous findings suggesting a preference for high-construal interpretations of behaviors (see Fujita, Henderson, Eng, Trope, \& Liberman, 2006; Rim, Hansen, \& Trope, 2013).

In sum, while Studies 2 and 3 demonstrated the effect of construal level on the vertical positioning of a stimulus, Study 4 exemplifies the reverse effect: The vertical positioning of a stimulus influences people's relative inclination to construe it at a high versus low level.

\section{General Discussion}

Many languages, including English, inherently assume an association between vertical position and construal level, as seen in terms such as high-construal or superordinate for abstract concepts or categories, and low-construal or subordinate for concrete concepts or exemplars. In the current research, we empirically examined the existence of association between vertical position and construal level and its bi-directional effects.

As expected, in Study 1 participants demonstrated both an implicit association between the two dimensions, as reflected in their performance on an SPF task, and an explicit association between them, as reflected in self-reports. In Studies 2 and 3, participants showed that they regarded it as intuitively more correct to position an abstract concept or superordinate category (high-construal level) at the top of a display and a concrete concept or exemplar (low-construal level) at the bottom of a display compared with the reverse. In Study 4, we manipulated the vertical position of stimuli and examined the effects on their construal. We found that participants preferred high-level construals of actions to a greater extent when these were presented at the top of a display than when they were presented at the bottom.

\section{Relation to Previous Findings}

Our findings are in accordance with those of Van Kerckhove et al. (2014), who showed that continuous activation of certain motor programs (raising or lowering the head and eyes) results in activation of high- versus low-construal levels. Indeed, the effects we observed in Study 4 may have been a product not only of the relative positioning of our stimuli on a vertical card, but also by a mild bodily feedback effect, driven by the fact that participants had to raise or lower their eyes very slightly to view the stimuli. Note, however, that unlike in the studies of Van Kerckhove et al., our manipulation involved only minor and momentary bodily feedback, as the positioning of stimuli was manipulated within the framework of a small display and within rather than between participants. Furthermore, bodily feedback is not likely to have contributed to the results of Studies 2 and 3 , as the concepts in those studies were presented, and hence processed, at the center of the display. Finally, the results of Study 1 support the existence of a mental association between relative vertical position and construal level, thus suggesting another mechanism (besides bodily feedback) underlying the effects found in the current research.

Interestingly, our findings join novel findings which attest to a possible association between vertical positioning of the self and construal level. Specifically, it has been shown that participants who experience themselves in a higher position (e.g., scanning the view from a mountaintop; imagining themselves on a higher floor; looking down from the top of a descending staircase) used a higher construal level than those who experienced themselves in a lower position (e.g., looking at a building from below; imagining themselves in a cellar; looking up from the bottom of an ascending staircase; Aggarwal \& Zhao, 2014; Slepian, Masicampo, \& Ambady, 2015). Thus, perceptions of either an external stimulus or one's own perspective as being higher (compared to lower) seem to result in use of a higher construal level. 


\section{Limitations}

One limitation of our studies is that in each study, each trial involved a built-in contrast between high-level and lowlevel construals. Future research should examine whether the results of our Studies 2 and 3 hold even when (for example) participants are presented with only one item (either an abstract concept or a concrete concept; either a category or an exemplar) in each trial.

In addition, the design of Study 4 did not include a control condition where the behavior and its descriptions were presented at the center of the card. Therefor, at this point we cannot tell whether our findings are driven mainly the positioning of the set at the top of the card, its positioning at the bottom of the card, or both.

\section{Implications}

The results of Study 4 suggest that the relative vertical positioning of information affects people's preference for construing it at a high versus low level. This finding has potential implications for the design of displays and the presentation of printed material. For example, these results suggest that the placement of questionnaire items along the vertical dimension may affect participants' response patterns, with items appearing at the top of a screen or page eliciting responses based on a high construal of the object of interrogation, and items appearing at the bottom potentially more likely to induce a low-construal of the very same object.

Furthermore, previous research has shown that processing of a stimulus is faster (i.e., easier) when associated dimensions of the stimulus match (Chae \& Hoegg, 2013; Cian, Krishna, \& Schwarz, 2015; Deng \& Kahn, 2009; Meier \& Robinson, 2004). All four studies reported in this paper point to an association between the vertical positioning of a stimulus and the level at which people construe it. Our findings thus suggest that high-construal information may be easier to process when presented higher in a display and low-construal information when presented lower in a display. If this is true, its potential implications are numerous. For example, previous studies show that the ease with which a message is processed contributes to its persuasiveness (Amit, Wakslak, \& Trope, 2012; Briñol, Tormala, \& Petty, 2013; Lee \& Aaker, 2004). Our findings thus suggest that messages aimed at motivating readers to engage in a certain behavior may be easier to process and hence more convincing when the aims and goals of the behavior are presented higher up in the display and the means and manner of performing it are presented lower down, compared with the other way around.

\section{Conclusion}

We found that construal level is associated with the vertical dimension, such that a stimulus's construal level affects its preferred vertical positioning and vice versa. These results contribute to the literature on construal-level theory and may have implications for various domains such as learning and the design of displays and questionnaires.

\section{References}

Aggarwal, P., \& Zhao, M. (2014). Seeing the big picture: The effect of height on the level of construal. Journal of Marketing Research, 52, 120-133. https://doi.org/10.1509/jmr.12.0067

Amit, E., Algom, D., \& Trope, Y. (2009). Distance-dependent processing of pictures and words. Journal of Experimental Psychology: General, 138, 400-415. https://doi.org/10.1037/ a0015835

Amit, E., Wakslak, C., \& Trope, Y. (2012). The use of visual and verbal means of communication across psychological distance. Personality \& Social Psychology Bulletin, 39, 43-56. https://doi. org/10.1177/0146167212460282

Bar-Anan, Y., Liberman, N., \& Trope, Y. (2006). The association between psychological distance and construal level: Evidence from an Implicit Association Test. Journal of Experimental Psychology: General, 135, 609-622. https://doi.org/10.1037/ 0096-3445.135.4.609

Bar-Anan, Y., Nosek, B. A., \& Vianello, M. (2009). The sorting paired features task: A measure of association strengths. Experimental Psychology, 56, 329-343. https://doi.org/ 10.1027/1618-3169.56.5.329

Briñol, P., Tormala, Z. L., \& Petty, R. E. (2013). Ease and persuasion: Multiple processes, meanings, and effects. In C. Unkelbach \& R. Greifender (Eds.), The experience of thinking: How the fluency of mental processes influences cognition and behaviour (pp. 101-118). New York, NY: Psychology Press. http://psycnet.apa.org/record/2013-00420-007

Bruno, N., \& Cutting, J. E. (1988). Minimodularity and the perception of layout. Journal of Experimental Psychology: General, 117, 161-170. http://doi.org/10.1037/0096-3445.117.2.161

Brysbaert, M., Warriner, A. B., \& Kuperman, V. (2014). Concreteness ratings for 40 thousand generally known English word lemmas. Behavior research methods, 46, 904-911. https://doi. org/10.3758/s13428-013-0403-5

Chae, B., \& Hoegg, J. (2013). The future looks "right": Effects of the horizontal location of advertising images on product attitude. Journal of Consumer Research, 40, 223-238. https://doi.org/ 10.1086/669476

Cho, Y. S., \& Proctor, R. W. (2003). Stimulus and response representations underlying orthogonal stimulus-response compatibility effects. Psychonomic Bulletin \& Review, 10, 45-73. https://doi.org/10.3758/BF03196468

Cian, L., Krishna, A., \& Schwarz, N. (2015). Positioning rationality and emotion: Rationality is up and emotion is down. Journal of Consumer Research, 42, 632-651. https://doi.org/10.1093/jcr/ ucv046

Cutting, J. E., \& Vishton, P. M. (1995). Perceiving layout and knowing distances: The integration, relative potency, and contextual use of different information about depth. In W. Epstein \& S. Rogers (Eds.), Perception of space and motion (pp. 69-117). New York, NY: Academic Press. https://doi.org/ 10.1016/B978-012240530-3/50005-5 
Day, S. B., \& Bartels, D. M. (2004). Temporal distance, event representation, and similarity. Proceedings of the Annual Meeting of the Cognitive Science Society, 26, 274-278. http:// citeseerx.ist.psu.edu/viewdoc/summary?doi=10.1.1.386.2111

Deng, X., \& Kahn, B. E. (2009). Is your product on the right side? The "location effect" on perceived product heaviness and package evaluation. Journal of Marketing Research, 46, 725-738. https://doi.org/10.1509/jmkr.46.6.725

Elias, Y., Nussinson, R., \& Roccas, S. (2018). Values and vocational choice: The effect of temporal distance. European Journal of Social Psychology, 48, 93-99. https://doi.org/10.1002/ ejsp.2286

Faul, F., Erdfelder, E., Lang, A.-G., \& Buchner, A. (2017). GPower version 3.1.9.2 [Computer software]. Retrieved from http:// www.gpower.hhu.de/en.html

Frost, R., \& Plaut, D. (2005). The word-frequency database for printed Hebrew. Retrieved from http://word-freq.mscc.huji.ac.il

Fujita, K., Henderson, M., Eng, J., Trope, Y., \& Liberman, N. (2006). Spatial distance and mental construal of social events. Psychological Science, 17, 278-282. https://doi.org/10.1111/ j.1467-9280.2006.01698.x

Gibson, J. J. (1950). The perception of the visual world. Oxford, UK: Houghton Mifflin. http://psycnet.apa.org/record/1951-04286000

Greenwald, A. G., McGhee, D. E., \& Schwartz, J. K. L. (1998). Measuring individual differences in implicit cognition: The Implicit Association Test. Journal of Personality and Social Psychology, 74, 1464-1480. https://doi.org/10.1037/00223514.74.6.1464

Lee, A. Y., \& Aaker, J. L. (2004). Bringing the frame into focus: The influence of regulatory fit on processing fluency and persuasion. Journal of Personality and Social Psychology, 86, 205-218. https://doi.org/10.1037/0022-3514.86.2.205

Li, C. Y., \& Guo, K. (1995). Measurements of geometric illusions, illusory contours and stereo-depth at luminance and colour contrast. Vision Research, 35, 1713-1720. https://doi.org/ 10.1016/0042-6989(94)00212-5

Libby, L. K., Shaeffer, E. M., \& Eibach, R. P. (2009). Seeing meaning in action: A bidirectional link between visual perspective and action identification level. Journal of Experimental Psychology: General, 138, 503-516. https://doi.org/10.1037/ a0016795

Liberman, N., \& Trope, Y. (1998). The role of feasibility and desirability considerations in near and distant future decisions: A test of temporal construal theory. Journal of Personality and Social Psychology, 75, 5-18. https://doi.org/10.1037/00223514.75.1.5

Liberman, N., \& Trope, Y. (2008). The psychology of transcending the here and now. Science, 322, 1201-1205. https://doi.org/ 10.1126/science.1161958

Liviatan, I., Trope, Y., \& Liberman, N. (2008). Interpersonal similarity as social distance: Implications for perceptions of others' actions. Journal of Experimental Social Psychology, 44, 1256-1269. https://doi.org/10.1016/j.jesp.2008.04.007

Markman, A. B., \& Wisniewski, E. J. (1997). Similar and different: The differentiation of basic-level categories. Journal of Experimental Psychology: Learning, Memory, and Cognition, 23, 54-70. https://doi.org/10.1037/0278-7393.23.1.54

McCloskey, M., \& Zaragoza, M. (1985). Misleading postevent information and memory for events: Arguments and evidence against memory impairment hypotheses. Journal of Experimental Psychology: General, 114, 1-16. https://doi.org/ 10.1037/0096-3445.114.1.1

Meier, B. P., \& Robinson, M. D. (2004). Why the sunny side is up: Association between affect and vertical position. Psychological
Science, 15, 243-247. https://doi.org/10.1111/j.0956-7976. 2004.00659.x

Meyers-Levy, J., \& Zhu, R. (2007). The influence of ceiling height: The effect of priming on the type of processing that people use. Journal of Consumer Research, 34, 174-186. https://doi.org/ $10.1086 / 519146$

Nosek, B. A. (2005). Moderators of the relationship between implicit and explicit evaluation. Journal of Experimental Psychology: General, 134, 565-584. https://doi.org/10.1037/00963445.134.4.565

Ooi, T. L., Wu, B., \& He, Z. J. (2001). Distance determined by the angular declination below the horizon. Nature, 414, 197-200. https://www.nature.com/articles/35102562

Pansky, A., \& Tenenboim, E. (2011). Inoculating against eyewitness suggestibility via interpolated verbatim vs. gist testing. Memory \& Cognition, 39, 155-170. https://link.springer.com/article/ 10.3758/s13421-010-0005-8

Previc, F. H. (1990). Functional specialization in the lower and upper visual fields in humans: Its ecological origins and neurophysiological implications. Behavioral and Brain Sciences, 13, 559-566. https://doi.org/10.1017/S0140525X00080018

Previc, F. H. (1998). The neuropsychology of 3-D space. Psychological Bulletin, 124, 123-164. https://doi.org/10.1037/00332909.124.2.123

Previc, F. H., Declerck, C., \& de Brabander, B. (2005). Why your "head is in the clouds" during thinking: The relationship between cognition and upper space. Acta Psychologica, 118, 7-24. https://doi.org/10.1016/j.actpsy.2004.10.012

Rim, S., Hansen, J., \& Trope, Y. (2013). What happens why? Psychological distance and focusing on causes versus consequences of events. Journal of Personality and Social Psychology, 104, 457-472. https://doi.org/10.1037/a0031024

Rosch, E., Mervis, C. B., Gray, W. D., Johnson, D. M., \& BoyesBraem, P. (1976). Basic objects in natural categories. Cognitive Psychology, 8, 382-439. https://doi.org/10.1016/0010-0285 (76) $90013-X$

Slepian, M. L., Masicampo, E. J., \& Ambady, N. (2015). Cognition from on high and down low: Verticality and construal level. Journal of Personality and Social Psychology, 108, 1-17. https://doi.org/10.1037/a0038265

Trope, Y., \& Liberman, N. (2010). Construal-level theory of psychological distance. Psychological Review, 117, 440-463. https://doi.org/10.1037/a0018963

Vallacher, R. R., \& Wegner, D. M. (1987). What do people think they're doing? Action identification and human behavior. Psychological Review, 94, 3-15. https://doi.org/10.1037/0033295X.94.1.3

Vallacher, R. R., \& Wegner, D. M. (1989). Levels of personal agency: Individual variation in action identification. Journal of Personality and Social Psychology, 57, 660-671. https://doi. org/10.1037/0022-3514.57.4.660

Van Kerckhove, A., Geuens, M., \& Vermier, I. (2014). The floor is nearer than the sky: How looking up or down affects construal level. Journal of Consumer Research, 41, 1358-1371. https:// doi.org/10.1086/679309

Wakslak, C., \& Trope, Y. (2009). The effect of construal level on subjective probability estimates. Psychological Science, 20, 5258. https://doi.org/10.1111/j.1467-9280.2008.02250.x

Wakslak, C. J., Trope, Y., Liberman, N., \& Alony, R. (2006). Seeing the forest when entry is unlikely: Probability and the mental representation of events. Journal of Experimental Psychology: General, 135, 641-653. https://doi.org/10.1037/00963445.135.4.641

Warriner, A. B., Kuperman, V., \& Brysbaert, M. (2013). Norms of valence, arousal, and dominance for 13,915 English lemmas. 
Behavior Research Methods, 45, 1191-1207. https://doi.org/ 10.3758/s13428-012-0314-x

Yonas, A., Elieff, C. A., \& Arterberry, M. E. (2002). Emergence of sensitivity to pictorial depth cues: Charting development in individual infants. Infant Behavior and Development, 25, 495514. https://doi.org/10.1016/S0163-6383(02)00147-9

\section{History}

Received December 25, 2017

Revision received November 6, 2018

Accepted November 19, 2018

Published online February 27, 2019

\section{Acknowledgments}

Ravit Nussinson has previously published under the name Ravit LevySadot. Study 4 was conducted as part of a Masters thesis by Yaron Elias. We are grateful to Nira Liberman and Anna Izoucheev for their comments on an earlier version of the manuscript. We are grateful to Zohar Weinstein, Yifat Weiss, and Maya Zadka for their assistance in this work. We are grateful to Iggi Pritzjer for his help in programming. We are grateful to Barbara Grant, Jean Vermel, Etti Levran, and Meira Ben-Gad for their assistance in copyediting.

\section{Publication Ethics}

The authors declare that there are no potential conflicts of interest with respect to the research, authorship, and/or publication of this article.

\section{Authorship}

All authors contributed to the design of the studies. Yoav Bar-Anan programmed, ran, and analyzed the data of Study 1. Sari Mentser programmed, ran, and analyzed the data of Studies 2 and 3. Yaron Elias ran and analyzed the data of study 4. Ravid Nussinson wrote the first draft, and all authors revised the manuscript.

\section{Open Data/Materials}

This study was preregistered at AsPredicted.Org, https://aspredicted. org/blind.php?x=y8up7g. All datasets are available at https://osf.io/ ky25r/.

\section{Funding}

This research was supported by Israel Science Foundation grant 359/16.

\section{Ravit Nussinson}

Department of Education and Psychology

The Open University of Israel

Raanana 43107

Israel

ravitnu@openu.ac.il

\section{Appendix}

Table A1. Word pairs used in Study 2

\begin{tabular}{|c|c|c|}
\hline & Concrete & Abstract \\
\hline 1. & Hut & Prediction \\
\hline 2. & String & Theory \\
\hline 3. & Fish & Vision \\
\hline 4. & Phone & Education \\
\hline 5. & Basket & Concentration \\
\hline 6. & Pin & Custom \\
\hline 7. & Butter & Precedent \\
\hline 8. & Leaf & Process \\
\hline 9. & Tomato & Ponder \\
\hline 10. & Corridor & Hallucination \\
\hline 11. & Note & Thought \\
\hline 12. & Plank & Contemplation \\
\hline 13. & Taxicab & Strategy \\
\hline 14. & Curtain & Prophecy \\
\hline 15. & Wall & Atmosphere \\
\hline 16. & Tree & Meaning \\
\hline 17. & Bread & Feeling \\
\hline 18. & Pot & Listening \\
\hline 19. & Clock & Opinion \\
\hline 20. & Lip & Hypothesis \\
\hline 21. & Radio & Context \\
\hline 22. & Vase & Improvization \\
\hline 23. & Bag & Frequency \\
\hline 24. & Road & Forecast \\
\hline 25. & Keyboard & Logic \\
\hline 26. & Pillow & Respite \\
\hline 27. & Blanket & Meditation \\
\hline 28. & Leg & Concept \\
\hline 29. & Chair & Essence \\
\hline 30. & Table & Explanation \\
\hline
\end{tabular}

Notes. The concrete word is on the left and the abstract is on the right. In Study 2, we counterbalanced for the words sides. 